\title{
In silico Elucidation of Dihydroquinine Mechanism of Action against Toxoplasma
}

\author{
gondii
}

\section{Joseph A. Ayariga ${ }^{2,5}$, Aarin M. Huffman', Audrey Napier², BK Robertson ${ }^{2,4}$, Daniel A. Abugri ${ }^{2,4,5^{*}}$}

${ }^{1}$ Department of Biology, College of Arts and Sciences, Tuskegee University, Tuskegee, AL, 36088, USA, ${ }^{2}$ Department of Biological Sciences, ${ }^{4}$ Microbiology PhD Program, ${ }^{5}$ Laboratory of Ethnomedicine, Parasitology, and Drug Discovery, College of Science, Technology, Engineering and Mathematics, ${ }^{5}$ Biomedical Engineering Program, Alabama State University, Montgomery,

AL, 36104, USA

* Corresponding Author:

Daniel A. Abugri, Ph.D.

${ }^{2}$ Department of Biological Sciences, ${ }^{4}$ Microbiology PhD. Program, Laboratory of Ethnomedicine, Parasitology, and Drug Discovery, College of Science, Technology, Engineering and Mathematics (C-STEM), Alabama State University, 1627 Hall Street Montgomery, Alabama 36104

Email address: dabugri@alasu.edu;Tel:334-604-8158

\begin{abstract}
Dihydroquinine (DHQ), is a quinine-based compound with anti-malarial properties. However, little is known about its mechanism of action against $T$. gondii inhibition, which shares similar biology with Plasmodium spp. In order to explore DHQ activity as an inhibitor of $T$. gondii using in vitro assays, we first used an in silico approach to decipher its mechanisms of action based on previous knowledge about its disruption of nucleic acid and protein synthesis. An in silico study was performed on T. gondii parasite replication, transcriptional and translational machinery to decipher the binding potentials of DHQ to some top selected enzymes. We report for the first time, using an in silico analysis that showed that DHQ binds strongly to DNA gyrase, Calcium Dependent Protein Kinase 1 (CDPK 1), and prolyl tRNA synthetase and thus could affect DNA replication, transcriptional and translational activities in T. gondii. Also, we found DHQ to effectively bind to mitochondria detoxifying enzymes (i.e., superoxide dismutase (SOD), peroxidoxin, and Catalase (CAT)). In conclusion, DHQ could be a lead compound for the treatment of toxoplasmosis when successfully evaluated using in vitro and in vivo models to confirm its effectiveness and safety.
\end{abstract}

Keywords: Dihydroquinine, DNA Gyrase, tRNA Synthetase, SOD, peroxidoxin and Catalase, Calcium Dependent Protein Kinases, T. gondii 


\section{Introduction}

Zoonotic parasitic infections continue to cause serious public health, veterinary and socioeconomic predicament globally (Ben-Harari and Connolly, 2019; Pappas et al., 2009; Flegr et al., 2014; Dubey, 2010). For example, toxoplasmosis, the disease caused by $T$. gondii affects nearly $1 / 3$ of the human population worldwide (Pappas et al., 2009; Flegr et al., 2014). More worrisome, a recent study, revealed that over 35 to $76 \%$ of wild and domestic felids are infected with $T$. gondii (Montazeri et al., 2020). This suggests that its infection in man and animals are on the increase globally. To overcome these parasitic diseases, different strategies are required which include, management, prevention, and treatment with safe and effective chemical inhibitors. Although few drugs are available for the treatment of individuals (humans and animals) infected with T.gondii, they are faced with limitations such as toxicity, high cost, and more so, most drugs are ineffective in treating the latent stage (bradyzoite) that continuously persist in the brain (Shammaa et al., 2021; Secrieru et al., 2020; Angel et al., 2020; Shiojiri et al., 2019). Thus, there is an urgent call for the development of novel compounds and inhibitors against this parasite, and more importantly an in-depth analysis and identification of the specific targets for safe and effective druggability and treatment.

DHQ is a pharmaceutical impurity associated with quine production (Nontprasert et al., 1996). It has been reported to have anti-malarial properties ( Gorka et al., 2013; Achan et al., 2011; Sanchez et al., 2008; Nontprasert et al., 1996; Pukrittayakamee et al., 1997; Brossi et al., 1973; Fayer et al., 1972; Brossi et al., 1971; Polet and Barr, 1968). Furthermore, its mechanism of action has been predicted in Plasmodium species to target nucleic acid and protein synthesis (Brossi et al., 1973; Brossi et al., 1971). However, its mechanism of action against T. gondii, which shares a similar phylum with Plasmodium is yet to be investigated. 
Here, using computational analysis, we seek to assess the molecular mechanism of action of DHQ in T. gondii using selected replicative, transcriptional, translational, and mitochondria machinery such as DNA gyrase, CDPKs, MIF, RNA synthase, SOD, peroxidoxin, and Catalase.

\section{Author Summary}

Early deciphering of compounds' mechanism of action is crucial for drug discovery and development. This approach saves time, resources and provides an insight into the possible mechanism of action of compounds of interest before wet experimental work could be carried out. In this paper, we used in silico approach as a first point of deciphering the mechanism of DHQ, a quinine derivative, that has been reported to have anti-Plasmodium activity in vitro and in vivo. The prediction showed that DHQ binds strongly to very important replicative, transcriptional, translational, and mitochondrial-associated proteins. Thus, this process was carried out as the first approach to predict DHQ's possible mechanism of action against $T$. gondii before performing wet labs to gain insight into the compound's inhibitory activity in T. gondii growth in vitro.

\section{Results and Discussion}

\section{In silico Docking of DHQ with T. gondii Replicative and Translational Machinery}

Based on our cellular and biochemical assay results, we used in silico docking analysis to decipher whether DHQ could be targeting the following enzymes/receptors: DNA Gyrase, TgCDPK1, tRNAs, MMIF, ROP5B, and ROP5C. These proteins play crucial roles in the parasite's invasion, survival, and replication. Herein, we analyzed for the theoretical binding affinities between DHQ and the enzymes/receptors as well as their ligand-receptor interactions at the docking pocket. The docking analysis was performed using extracted crystalized structures of the receptors from the RCSB website or modeled using the online modeling software SWISS-MODEL 
(https://swissmodel.expasy.org/). DHQ, 3-Amino-1H-pyrazole-4-carboxamide, and Moxifloxacin were downloaded from the PubChem database (https://pubchem.ncbi.nlm.nih.gov/).

\section{DHQ interaction with $T$. gondii DNA Gyrase interactions}

To stop the progression of multidrug-resistant tuberculosis, fluoroquinolone has been shown to have high potency, and the mechanism of action of fluoroquinolone is known to target DNA gyrase (Blower et al., 2016). It has also been proven in parasites (e.g., Trypanosomes) that some quinolone drugs bind to DNA gyrase to form a complex that blocks transcription by RNA polymerase (Willmott et al., 1994). DNA gyrase of $T$. gondii has also been indicated as a potential target for drugs against T. gondii (Lin et al., 2015). While the crystal structure of the DNA gyrase of multidrug-resistant tuberculosis has been discovered, the DNA gyrase of $T$. gondii is currently unavailable. In this work, we used the crystal structure of multidrug-resistant tuberculosis as a model study receptor to dock DHQ as a ligand and to analyze for their binding affinities $(\Delta \mathrm{G})$. To also show if there exist any contrasting binding affinities between DHQ and fluoroquinolone for the $M$. tuberculosis DNA gyrase as well as $T$. gondii DNA gyrase, their binding affinities were compared and the specific amino acids interacting with these compounds were analyzed for the type of interactions occurring at the binding pocket. While fluoroquinolone (also known as moxifloxacin) showed a slightly lower binding affinity of $-7.2 \mathrm{kcal} / \mathrm{mol}$ (Table 1) to gyrase of $M$. tuberculosis, DHQ bonded to T. gondii (TgDNA) gyrase with a binding affinity of $-8.0 \mathrm{kcal} / \mathrm{mol}$ (Table 1) compared to DHQ - M. tuberculosis DNA gyrase interaction which produced a relative free binding energy of $-6.6 \mathrm{kcal} / \mathrm{mol}$ (Table 1, Figure 2A, 2B and 2E). As shown in Figure 1, the 3D rendition of DHQ in red color interacting with gyrase in green color, also in Figure 1, a 2D depiction of DHQ interacting with gyrase at the binding pocket indicates that DHQ forms a strong hydrogen bond with ARG234 producing a bond distance of $1.90 \AA$, for which DHQ acts as the 
hydrogen acceptor and ARG234 the donor. The ALA255 of TgDNA gyrase binds to DHQ via hydrophobic interactions, forming a pi-alkyl bond with DHQ with a bond distance of $4.69 \AA$ in the binding pocket. Forming a hydrogen bond at $2.97 \AA$ with DHQ, TgDNA gyrase via ARG234 acts as a hydrogen donor for which DHQ is the acceptor. DHQ was able to form a stable hydrogen bond with TgDNA gyrase via the gyrase's ASP200 amino acid residue which acts as a hydrogen acceptor. A bond distance of $1.99 \AA$ is established between them. ARG195 interacts with DHQ via hydrogen bond also, for which ARG195 is the hydrogen acceptor. LEU257 of TgDNA gyrase establishes pi-sigma interaction with DHQ to establish a hydrophobic bond of distance $3.38 \AA$. Other hydrophobic bonds formed by TgDNA gyrase with DHQ established in the binding pocket includes amino acids ALA203 (bond distance, $3.55 \AA$ A), ALA255 (bond distance, $5.26 \AA$ A), LEU257 (bond distance, $5.32 \AA$ ), ALA267 (bond distance, $4.72 \AA$ ), LEU230 (bond distance, $4.94 \AA$ ), LEU257 (bond distance, $4.62 \AA$ ) and ALA255 (bond distance, $4.69 \AA$ ). This analysis points to stable DHQ-DNA gyrase interactions, illustrating the possible mechanism of action of DHQ as a T. gondii inhibitor via the inhibition of DNA replication through the binding to and interfering in gyrase activity during the parasite's DNA replication. This knowledge hints at a possible pharmacological utility of DHQ against $T$. gondii and multidrug-resistant tuberculosis.

\section{DHQ and $T$. gondii prolyl tRNA synthetase interactions}

Prolyl-tRNA synthetase belongs to the aminoacyl-tRNA synthetase family and plays a crucial role in protein translation in living cells. The tRNA synthetase of $T$. gondii has been a target for several compounds used as therapeutics against T. gondii and other Apicomplexans, e.g., the use of febrifugine and halofuginone (Mishra et al., 2019). This current work revealed that DHQ targets the prolyl tRNA synthetase of $T$. gondii with a high relative free binding energy of $-7.7 \mathrm{kcal} / \mathrm{mol}$ (Table 1). Figure 3C is the 3D depiction of the DHQ interacting with the tRNA synthetase of $T$. 
gondii. Also illustrated in Figure 3C and Figure 3D), a 2D illustration of the contacts of DHQ at the binding site to tRNA synthetase of $T$. gondii indicates that DHQ shares two hydrogen bonds at amino acids ARG594, and GLN555. There exists also a van der Waals interaction between the compound and the amino acid HIS560, a pi-alkyl interaction at amino acid PHE415 and pi-cation interaction with ARG470.

This analysis shows that there is a stable DHQ- prolyl tRNA synthetase interactions, pointing to the capacity of DHQ as $a T$. gondii inhibitor by interrupting the $T$. gondii protein translational activity by binding to the parasite's prolyl tRNA synthetase.

\section{DHQ and Macrophage Migration Inhibitory Factor of $T$. gondii (MIF) Interactions}

MIF is a proinflammatory molecule in mammals with homologs in parasites that possesses tautomerase and oxidoreductase enzymatic activities (Sommerville et al., 2013). Here we downloaded the tautomerase active MIF of $T$. gondii and studied the binding of DHQ to this molecule. Even though similar structural homology of $T$. gondii and mammalian MIFs do exist, their respective tautomerase active sites are different, and this justifies the variation in inhibitor sensitivity (Sommerville et al., 2013). We showed that T. gondii MIF binds stably to DHQ at a binding affinity of $-6.9 \mathrm{kcal} / \mathrm{mol}$ (Figure 3A). As shown in (Figure 3A), DHQ is bound to two loops of the MIF molecule. The 2D rendering (Figure 3B) shows that the most stable conformation was achieved by the binding of DHQ to the PHE114, PRO1, ILE65, LYS33, TYR37, and PRO34 via hydrogen bonding, alkyl, pi-alkyl, pi-cation, pi-pi stacked, and alkyl interactions, respectively. Since MIF of $T$. gondii is known to play an immunomodulatory role during the $T$. gondii infection process (Sommerville et al., 2013), this stable binding of DHQ to MIF interferes and deregulates the parasite's MIF proper function. 


\section{DHQ and ROP5B/C (T. gondii virulent allele) pseudokinase interactions}

ROP5B/C belongs to the ROP5 family which is a set of polymorphic pseudokinases. They have been demonstrated to play major roles in $T$. gondii pathogenesis (Reese et al., 2011; Reese et al., 2014). They play critical functions in subcellular localization of specific receptors, recognition of partner molecules, and regulators of signaling networks (Boudeau et al., 2006). In this study, we investigated the possibility of DHQ dysregulation of ROP5B by the compound binding to the pseudokinase and hindering its proper functional conformations. Our docking analysis showed that ROP5B interacted with DHQ with a high binding affinity of $-6.9 \mathrm{kcal} / \mathrm{mol}$. In (Figure 4A), DHQ in red color is shown to bind to ROP5B (green).

Also, the 2D depiction (Figure 4B) of the contacts of DHQ at the binding site to ROP5B pseudokinase of $T$. gondii shows DHQ interacting with amino acids ARG245, and GLU275 via pi-cation/anion interactions. DHQ established pi-pi-T shaped interaction with the pseudokinase at PHE265. Also, at LEU279, there was a pi-sigma bond with the compound. The ROP5C pseudokinase (Reese et al., 2011), on the other hand, showed a binding affinity of $-7.4 \mathrm{kcal} / \mathrm{mol}$ (Table 1) with DHQ. DHQ is shown in Figure 4C to interact with ROP5C, and in Figure 4D, the 2D diagram shows a stable interaction at the binding pocket in which LYS263 of ROP5C makes hydrogen bond contact with the compound, VAL248 makes a pi-sigma contact, whereas ARG241 makes an alkyl contact with DHQ. MET337 however, makes a pi-sulfur bond with DHQ. Collectively, these interactions create stable and strong bonds between DHQ and ROP5C, pointing to the capability of DHQ as a T. gondii inhibitor by the mechanism of binding to and disrupting the $T$. gondii pathogenesis process.

\section{DHQ and Calcium-Dependent Protein Kinase 1 from $T$. gondii (TgCDPK1) interactions.}


The CDPK1 enzyme in $T$. gondii controls multiple processes that are crucial for the parasite's intracellular replicative cycle and the parasite's secretion of adhesins (Ojo et al., 2010; Johnson et al., 2012). This enzyme plays a major function in the ability of the parasite to invade host cells, move, and egress (Fluorentine et al., 2017). We analyzed how effective DHQ binds to TgCDPK1 and thus affects the replicative and invasion properties of the parasite. Interestingly, the binding affinity of DHQ to TgCDPK1 was $-6.7 \mathrm{kcal} / \mathrm{mol}$, a $-1.2 \mathrm{kcal} / \mathrm{mol}$ higher than a known inhibitor of TgCDPK1 called 3-Amino-1H-pyrazole-4-carboxamide which recorded $-5.5 \mathrm{kcal} / \mathrm{mol}$. As shown in (Figure 5A, and Figure 5B) the ligand-receptor interaction showed that DHQ interacts with TgCDPK1 via two hydrogen bonds emanating from LEU345 and ARG439, two alkyl bonds formed between Lys59, LYS435, and LEU438, and finally a pi-anion interaction between GLU64 and DHQ. The $-5.5 \mathrm{kcal} / \mathrm{mol}$ of 3-Amino-1H-pyrazole-4-carboxamide interaction with TgCDPK1 came from the binding of the compound to the active site with the following residues: ILE212, LYS338, GLN334, GLN341, and GLU178 which all demonstrated conventional hydrogen bonding with the compound except GLU178 which established a pi-anion bond with 3-Amino1H-pyrazole-4-carboxamide (Figure 5C, and 5D).

LYS338 however, shows a strong unfavorable donor-donor interaction with the hydrogen moiety of 3-Amino-1H-pyrazole-4-carboxamide. This interaction therefore might have caused a considerable loss of affinity of 3-Amino-1H-pyrazole-4-carboxamide to TgCDPK1 compared to DHQ. Hence, the strong unfavorable donor-donor interaction recorded seems to slightly dampen this stability of 3-Amino-1H-pyrazole-4-carboxamide to TgCDPK1 interaction, hence the recorded $-5.5 \mathrm{kcal} / \mathrm{mol}$ binding affinity, DHQ on the other hand which produced a very high affinity of $-8 \mathrm{kcal} / \mathrm{mol}$ recorded no unfavorable bonds in its binding pocket. Hence, we predict a higher inhibitory potential for DHQ on TgCDPK1 than 3-Amino-1H-pyrazole-4-carboxamide. 
The binding of DHQ or 3-Amino-1H-pyrazole-4-carboxamide to TgCDPK1 prevents T. gondii phosphorylation and ATP binding activities (Ravi et al., 2020).

\section{DHQ interaction with T. gondii Mitochondria proteins (SODs, Peroxiredoxins, and Catalase)}

Mitochondria is a well-known important energy organelle in eukaryotes and especially, apicomplexans parasites e.g., T. gondii. The organelle's basic functions include cytosolic nucleic iron-sulfur proteins detoxification, nucleic acid synthesis, fatty acid synthesis, and protein synthesis as well as generation of energy for cellular mechanistic activities (Syn et al., 2017; Lill et al., 2014; Kwok et al., 2004; Melo et al., 2000). Thus, cellular activities that cause mitochondria imbalances could lead to disruption of the above functions mentioned as well as the membrane potential in T. gondii.

For instance, the common causes of mitochondria imbalances in the cell could be partly due to the induction of external and internal stimuli such as stress, chemical inducers, and microbial infections (Syn et al., 2017; Kwok et al., 2004; Callahan et al., 1988; McGonigle and Dalton,1998; Nathan and Shiloh, 2000). The inducers of mitochondria function often cause the excessive generation of reactive oxygen species (ROS) (e.g., hydrogen peroxide, superoxide radical, and hydroxyl radical) by parasites (Kwok et al., 2004; Vercesi et al., 1998). It has been well documented that this ROS creates microenvironmental oxidative stress intracellularly which causes toxicity to parasites and eventually parasites death (Kwok et al., 2004). To overcome this ROS toxicity in eukaryotic and especially parasites cells, the mitochondria use its internal enzymes such as superoxide dismutase, catalase, glutathione peroxidase, and peroxiredoxins to form an antioxidant to detoxify the reactive oxygen species (ROS) (Kwok et al., 2004; Son et al., 2001; 
Odberg-Ferragut et al., 2000; Ding et al., 2000; Kaasch and Joiner, 2000; Vercesi et al., 1998; Miller and Britigan, 1997).

To validate whether DHQ had any way of counteracting the mitochondrial enzymes that aid $T$. gondii to detoxify drugs and other induces of ROS, we screen DHQ against the above-mentioned enzymes. Interestingly, DHQ interaction with T. gondii peroxidoxin (PRX), SOD3, and CAT had binding affinities of -7.0, -6.9, -6.8 kcal/mol, respectively. Figure 6A and 6B depicts DHQ (red) bound to the SOD3 molecule in the binding pocket. As shown in Figure 6B, DHQ establishes a stable hydrogen bond with GLU210. It also interacts with SOD3 via alkyl and pi-alkyl hydrophobic interactions through amino acids HIS80, TYR84, PHE168, and ARG221. Other weaker van der Waals interactions established between SOD3 and DHQ were recorded on amino acids LYS166, GLY169, ASN87, THR165, HIS167, TRP 209, HIS211, ASN 219, ASP220, and GLY222. Together these interactions enhanced the stability of the binding of DHQ to the SOD3 molecule. Figures 6C and 6D depict the interaction of DHQ (red) with the SOD2 molecule. As shown in Figure 6C, DHQ formed a stable hydrogen bond with ALA118 and HIS115. It also interacts with SOD2 via alkyl and pi-alkyl hydrophobic interactions through amino acids LYS122, TYR119, ARG260, and PRO261. The weak van der Waals interactions established between SOD2 and DHQ were recorded on amino acids ASN151, ALA203, PHE206, GLY207, TRP248, GLU249, ASN258, and ASP259. Accumulatively these interactions boosted the stability of the biding of DHQ to SOD2 molecule.

Figure 7A and 7B depicts DHQ (red) bound to Tg Catalase chain A. As depicted in Figure 7B, DHQ forms a stable hydrogen bond with HIS183 and shares a pi-sigma bond with VAL291 and VAL443 respectively. VAL291 and VAL443 can also interact with DHQ via alkyl hydrophobic interactions. Tg Catalase also can bind to DHQ via its PRO293 by another hydrophobic alkyl 
interaction with the hydroxyl group of the DHQ. Figures 7C and 7D depict the interaction of DHQ (red) with the Tg peroxidoxin molecule. As shown in Figure 7D, Tg peroxiredoxin binds tightly to DHQ via two hydrogen bonds established between DHQ at amino acids AS204 and TYR164. It is also able to stabilize DHQ in the binding pocket via hydrophobic interactions such as pi-pi-T shaped interactions, alkyl, and pi-alkyl interactions through the amino acids TYR164, IL3106, VAL206, LEU128, PHE132, LEU228, and PRO 127. Also, a weaker van der Waal force exists between ALA163, SER188, LEU190, THR131, and ALA269 with DHQ. These altogether stabilized DHQ in Tg peroxiredoxin binding pocket.

Our in-silico studies, corroborated with the mechanism(s) of action of DHQ previously reported in Plasmodium species to inhibit DNA replication, RNA, and protein synthesis (Achan et al., 2011; Nontprasert et al., 1996; Brossi et al., 1973; Brossi et al., 1971; Polet and Barr, 1968).

It has been well documented that in multidrug-resistant (MDR) tuberculosis treatment, quinolone-based drugs such as fluoroquinolone can effectively inhibit the bacteria by targeting its DNA gyrase (Blower et al., 2016). Another study in Trypanosomes has also elucidated that DNA gyrase perfectly binds with quinolone drugs thus forming a unique complex that blocks transcription at the RNA polymerase site (Willmott et al., 1994). Thus, DHQ might be targeting the DNA gyrase of $T$. gondii which has been identified as a potential target for drugs against $T$. gondii (Blower et al., 2016). Additionally, the observed effective and stable DHQ-prolyl tRNA synthetase interactions obtained from the docking studies point to the possibility of DHQ inhibiting $T$. gondii replication and invasion by interrupting the $T$. gondii protein translational activity by strongly binding to the parasite's prolyl tRNA synthetase.

Calcium-dependent protein kinases are very important to T. gondii invasion and survival processes (Ojo et al., 2010; Johnson et al., 2012). Interestingly, our docking studies showed that 
DHQ or 3-Amino-1H-pyrazole-4-carboxamide effectively binds to TgCDPK1 and thus could avert its phosphorylation and ATP binding activities (Blower et al., 2016). The anti-proliferation and anti-invasion results obtained in the in vitro studies corroborated with our in silico docking studies that indicate that DHQ effectively binds to TgCDPKI. In previous studies, by (Ojo et al., 2010; Johnson et al., 2012), TgCDPKI has been discovered to play a crucial role in $T$. gondii proliferation and invasion of host cells. Thus, the strong binding affinity obtained implies that DHQ could block TgCDPKI expressions and hence blocks invasion and prevent the proliferation of T. gondii.

The T. gondii peroxidoxin (PRX), SOD3, and CAT's strong interaction with DHQ observed, suggested that DHQ could be an excellent inhibitor of the $T$. gondii mitochondria ROS detoxifying enzymes. Thus, could cause parasites death due to excessive toxicity of the generated ROS caused by the immune-related cells to offset any microbial infection and chemical inducers such as drugs (Syn et al., 2017; Kwok et al., 2004; Callahan et al., 1988; McGonigle and Dalton,1998; Nathan and Shiloh, 2000). However, since this is in silico, further studies are needed to confirm this conjecture.

\section{Conclusion}

DHQ was highly effective in binding to replicative, transcriptional, and translational machinery of $T$. gondii in silico. Thus, could cause inhibition of $T$. gondii growth, invasion, and egress. Also, based on the in-silico data obtained regarding mitochondria machinery association with DHQ, we believed that the compound might cause ROS generation in $T$. gondii tachyzoites and eventually mitochondrial membrane disruption. All these predictions will need further investigation using in vitro experiments. 


\section{Materials and Methods}

\section{Molecular Docking of $\mathrm{DHQ}$}

The protein-ligand docking approach was employed to analyze and identify the specific amino acid interactions between DHQ and the respective receptors found on T. gondii. The binding affinities afford a good prediction of the ability of DHQ to inhibit $T$. gondii via various mechanisms modulated by proteins/enzymes (DNA gyrase, Calcium Dependent Protein Kinase 1 (CDPK 1), and prolyl tRNA synthetase) understudy.

The following platforms were used for the computational studies of the effect of DHQ on various replicative, and translational machinery; Vina Autodock, Pymol (2) (Schrodinger, LLC), PyRx, and Discovery Studio 2021.

\section{TgDNA gyrase structure modeling}

Currently, the TgDNA gyrase crystal structure is not available. Therefore, an in silico model was made using the TgDNA sequence. In summary, the TgDNA gyrase subunit B sequence (ID KFG61327.1) was downloaded from the NCBI website. The sequence was converted into FASTA format using the sequence format converter (http://avermitilis.ls.kitasato-u.ac.jp/readseq.cgi), afterward, the sequence was modeled into its predicted 3D structure using the online SWISSMODEL tool (https://swissmodel.expasy.org/). The model with the highest score in Global Model Quality Estimation (GQME) was utilized for the binding study.

\section{Ligand preparation}


The DHQ was prepared as a ligand for docking onto the following receptors, 4DH4, ROP5C, ROP5B, and enzymes (TgDNA gyrase, Calcium Dependent Protein Kinase 1 (CDPK 1), and prolyl tRNA synthetase). These receptors/enzymes were selected for the molecular docking analysis to confirm the in vitro mechanism of action(s) based on their association with replication, invasion, and survival of T. gondii. Additionally, previous works on Plasmodium spp implicated dihydroquinine to inhibit DNA, RNA, and protein synthesis (Brossi et al., 1973; Brossi et al., 1971; Polet and Barr, 1968). The chemical structure of DHQ was extracted from the PubChem database. The ligand was uploaded into PyRx software via the Open babel plugin, and the 3D and geometry optimizations with energy minimization of DHQ structure were carried out. DHQ structure was converted to autodock DHQ.pdbqt ligand file via the same Open babel plugin in PyRx. The Vina wizard was used to load the DHQ.pdbqt file as a ligand to the respective receptors/enzymes used in the docking process. Huang et al. (2015), demonstrated that 5-aminopyrazole-4-carboxamide derivatives were selectively potent inhibitors of 4YJN (Calcium-Dependent Protein Kinase 1 (TgCDPK1) from T. gondii. They also showed the effectiveness of the structure-activity relationship of this compound in $a$ mouse model against $T$. gondii in the brain, spleen, and peritoneal fluids. In this computational investigation, we analyzed the binding affinity of 5aminopyrazole-4-carboxamide also to 4YJN to serve as an experimental control for all the binding studies of DHQ on the various molecules. The 2D structure (PDB format) of 5-aminopyrazole-4carboxamide was downloaded from the PubChem database (CID_79254) and processed for docking. 5-aminopyrazole-4-carboxamide was prepared for docking following the same process as started earlier. 


\section{Preparation of protein structures and grid generation}

The Crystal structures of virulent alleles ROP5B (3Q5Z) (Reese and Boothroyd, 2011; Reese et al., 2014), and ROP5C (4VL8) (Reese et al., 2014) of T. gondii were downloaded from the RCSB homepage (http://www.rscb.org) at a resolution of $1.90 \AA$ (Reese and Boothroyd, 2011), and $1.72 \AA$ A, respectively. Also, 4DH4 (Sommerville et al., 2013), 4YJN (Cardew et al., 2018), and 6A88 (Mishra et al., 2019) molecules were extracted from the same website at resolutions of 1.82 $\AA$, $2.60 \AA$, and $2.60 \AA$, respectively. These proteins were loaded into Pymol 2 (Schrodinger, LLC), for detailed analysis of proteins' structural elements, then each molecule was exported in a PDB file format with the removal of water molecules. Vina Autodock was used to adjust charges, check, and replace missing atoms and add polar hydrogens to the proteins structures. Next, all protein structures were energy minimized using the Autodock Vina. Additionally, proteins were loaded into PyRx and converted to receptors, the receptor grid boxes were generated in PyRx using the built-in Vina Wizard module, boxes were maximized to cover all active sites of the receptors. Docking of compounds to their respective receptors was achieved using the AutoDock tool in PyRx and analysis of specific amino acids in the binding pocket with DHQ or other ligands were achieved using Discovery Studio 2021.

\section{AUTHOR CONTRIBUTIONS}

DAA conceived the idea. JAA performed the in-silico studies. JAA analyzed the data and drafted the first manuscript under the supervision of DAA. DAA, AN, and BK corrected the original manuscript. All authors approved the final manuscript.

\section{FUNDING}

No external funding was provided. DAA had internal funding from the Department of Biological Sciences at Alabama State University (ASU) to perform this work. 


\section{ACKNOWLEDGMENTS}

None

\section{Supplementary Material}

Supplementary Materials have been included in the main manuscript.

\section{Data Availability Statement}

The datasets generated for this study are available upon request from the corresponding author.

\section{REFERENCES}

1. Vercesi AE, Rodrigues CO, Uyemura SA, Zhong L, Moreno SN. Respiration and oxidative phosphorylation in the apicomplexan parasite Toxoplasma gondii. Journal of Biological Chemistry. 1998 Nov 20;273(47):31040-7.

2. Miller RA, Britigan BE. Role of oxidants in microbial pathophysiology. Clinical microbiology reviews. 1997 Jan;10(1):1-8.

3. Pukrittayakamee S, Looareesuwan S, Keeratithakul D, Davis TM, Teja-Isavadharm P, Nagachinta B, Weber A, Smith AL, Kyle D, White NJ. A study of the factors affecting the metabolic clearance of quinine in malaria. European journal of clinical pharmacology. 1997 Sep;52(6):487-93.

4. Callahan HL, Crouch RK, James ER. Helminth anti-oxidant enzymes: a protective mechanism against host oxidants?. Parasitology Today. 1988 Aug 1;4(8):218-25.

5. Ding M, Clayton C, Soldati D. Toxoplasma gondii catalase: are there peroxisomes in toxoplasma?. Journal of cell science. 2000 Jul 1;113(13):2409-19.

6. Kaasch AJ, Joiner KA. Targeting and subcellular localization of Toxoplasma gondii catalase: Identification of peroxisomes in an apicomplexan parasite. Journal of Biological Chemistry. 2000 Jan 14;275(2):1112-8. 
7. McGonigle S, Dalton JP, James ER. Peroxidoxins: a new antioxidant family. Parasitology Today. 1998 Apr 1;14(4):139-45.

8. Nathan C, Shiloh MU. Reactive oxygen and nitrogen intermediates in the relationship between mammalian hosts and microbial pathogens. Proceedings of the National Academy of Sciences. 2000 Aug 1;97(16):8841-8.

9. Ödberg-Ferragut C, Renault JP, Viscogliosi E, Toursel C, Briche I, Engels A, Lepage G, Morgenstern-Badarau I, Camus D, Tomavo S, Dive D. Molecular cloning, expression analysis and iron metal cofactor characterisation of a superoxide dismutase from Toxoplasma gondii. Molecular and biochemical parasitology. 2000 Feb 25;106(1):121-9.

10. Son ES, Song KJ, Shin JC, Nam HW. Molecular cloning and characterization of peroxiredoxin from Toxoplasma gondii. The Korean journal of parasitology. 2001 Jun;39(2):133.

11. Kwok LY, Schlüter D, Clayton C, Soldati D. The antioxidant systems in Toxoplasma gondii and the role of cytosolic catalase in defence against oxidative injury. Molecular microbiology. 2004 Jan;51(1):47-61.

12. Achan J, Talisuna AO, Erhart A, Yeka A, Tibenderana JK, Baliraine FN, Rosenthal PJ, D'Alessandro U. Quinine, an old anti-malarial drug in a modern world: role in the treatment of malaria. Malaria journal. $2011 \mathrm{Dec} ; 10(1): 1-2$.

13. Angel SO, Vanagas L, Ruiz DM, Cristaldi C, Saldarriaga Cartagena AM, Sullivan Jr WJ. Emerging Therapeutic Targets Against Toxoplasma gondii: Update on DNA Repair Response Inhibitors and Genotoxic Drugs. Frontiers in Cellular and Infection Microbiology. 2020 Jun 12;10:289. 
14. Ben-Harari RR, Connolly MP. High burden and low awareness of toxoplasmosis in the United States. Postgraduate medicine. 2019 Feb 17;131(2):103-8.

15. Blower TR, Williamson BH, Kerns RJ, Berger JM. Crystal structure and stability of gyrasefluoroquinolone cleaved complexes from Mycobacterium tuberculosis. Proceedings of the National Academy of Sciences. 2016 Feb 16;113(7):1706-13.

16. Brossi A, Uskoković M, Gutzwiller J, Krettli AU, Brener Z. Antimalarial activity of racemic 3-Epi dihydroquinine, 3-Epi dihydroquinidine and their various racemic analogs in mice infected with Plasmodium berghei. Experientia. 1973 Mar;29(3):367-8.

17. Brossi A, Uskoković M, Gutzwiller J, Krettli AU, Brener Z. Antimalarial activity of natural, racemic and unnatural dihydroquinine, dihydroquinidine and their various racemic analogs in mice infected with Plasmodium berghei. Experientia. 1971 Sep;27(9):1100-1.

18. Cardew EM, Verlinde CL, Pohl E. The calcium-dependent protein kinase 1 from Toxoplasma gondii as target for structure-based drug design. Parasitology. 2018 Feb;145(2):210-8.

19. Dubey JP. Toxoplasmosis of animals and humans. CRC press; 2016 Apr 19.

20. Dwyer DJ, Kohanski MA, Hayete B, Collins JJ. Gyrase inhibitors induce an oxidative damage cellular death pathway in Escherichia coli. Molecular systems biology. 2007;3(1):91.

21. Fayer R, Melton ML, Sheffield HG. Quinine inhibition of host cell penetration by Toxoplasma gondii, Besnoitia jellisoni, and Sarcocystis sp. in vitro. The Journal of parasitology. 1972 Jun 1:595-9.

22. Flegr J, Prandota J, Sovičková M, Israili ZH. Toxoplasmosis-a global threat. Correlation of latent toxoplasmosis with specific disease burden in a set of 88 countries. PloS one. 2014 Mar 24;9(3):e90203. 
23. Rutaganira FU, Barks J, Dhason MS, Wang Q, Lopez MS, Long S, Radke JB, Jones NG, Maddirala AR, Janetka JW, El Bakkouri M. Inhibition of calcium dependent protein kinase 1 (CDPK1) by pyrazolopyrimidine analogs decreases establishment and reoccurrence of central nervous system disease by Toxoplasma gondii. Journal of medicinal chemistry. 2017 Dec 28;60(24):9976-89.

24. Gorka AP, Sherlach KS, de Dios AC, Roepe PD. Relative to quinine and quinidine, their 9epimers exhibit decreased cytostatic activity and altered heme binding but similar cytocidal activity versus Plasmodium falciparum. Antimicrobial agents and chemotherapy. 2013 Jan;57(1):365-74.

25. Huang W, Ojo KK, Zhang Z, Rivas K, Vidadala RS, Scheele S, DeRocher AE, Choi R, Hulverson MA, Barrett LK, Bruzual I. SAR studies of 5-aminopyrazole-4-carboxamide analogues as potent and selective inhibitors of Toxoplasma gondii CDPK1. ACS medicinal chemistry letters. 2015 Dec 10;6(12):1184-9.

26. Johnson SM, Murphy RC, Geiger JA, DeRocher AE, Zhang Z, Ojo KK, Larson ET, Perera BG, Dale EJ, He P, Reid MC. Development of Toxoplasma gondii calcium-dependent protein kinase 1 (Tg CDPK1) inhibitors with potent anti-toxoplasma activity. Journal of medicinal chemistry. 2012 Mar 8;55(5):2416-26.

27. Ojo KK, Larson ET, Keyloun KR, Castaneda LJ, DeRocher AE, Inampudi KK, Kim JE, Arakaki TL, Murphy RC, Zhang L, Napuli AJ. Toxoplasma gondii calcium-dependent protein kinase 1 is a target for selective kinase inhibitors. Nature structural \& molecular biology. 2010 May;17(5):602-7. 
28. Lill R, Srinivasan V, Mühlenhoff U. The role of mitochondria in cytosolic-nuclear ironsulfur protein biogenesis and in cellular iron regulation. Current opinion in microbiology. 2014 Dec 1;22:111-9.

29. Lin TY, Nagano S, Heddle JG. Functional analyses of the Toxoplasma gondii DNA gyrase holoenzyme: a janus topoisomerase with supercoiling and decatenation abilities. Scientific reports. 2015 Sep 28;5(1):1-3.

30. Melo EJ, Attias M, De Souza W. The single mitochondrion of tachyzoites of Toxoplasma gondii. Journal of structural biology. 2000 May 1;130(1):27-33.

31. Mishra S, Malhotra N, Kumari S, Sato M, Kikuchi H, Yogavel M, Sharma A. Conformational heterogeneity in apo and drug-bound structures of Toxoplasma gondii prolyl-tRNA synthetase. Acta Crystallographica Section F: Structural Biology Communications. 2019 Nov 1;75(11):714-24.

32. Montazeri M, Galeh TM, Moosazadeh M, Sarvi S, Dodangeh S, Javidnia J, Sharif M, Daryani A. The global serological prevalence of Toxoplasma gondii in felids during the last five decades (1967-2017): a systematic review and meta-analysis. Parasites \& vectors. 2020 Dec;13(1):1-0.

33. Nontprasert A, Pukrittayakamee S, Kyle DE, Vanijanonta S, White NJ. Antimalarial activity and interactions between quinine, dihydroquinine and 3-hydroxyquinine against Plasmodium falciparum in vitro. Transactions of the Royal Society of Tropical Medicine and Hygiene. 1996 Sep 1;90(5):553-5.

34. Pappas G, Roussos N, Falagas ME. Toxoplasmosis snapshots: global status of Toxoplasma gondii seroprevalence and implications for pregnancy and congenital toxoplasmosis. International journal for parasitology. 2009 Oct 1;39(12):1385-94. 
35. Polet H, Barr CF. Chloroquine and dihydroquinine. In vitro studies of their antimalarial effect upon Plasmodium knowlesi. Journal of Pharmacology and Experimental Therapeutics. 1968

Dec 1;164(2):380-6.

36. Jain R, Gupta S, Munde M, Pati S, Singh S. Development of novel anti-malarial from structurally diverse library of molecules, targeting plant-like CDPK1, a multistage growth regulator of $P$. falciparum. Biochemical Journal. 2020 May 29;477(10):1951-70.

37. Reese ML, Boothroyd JC. A conserved non-canonical motif in the pseudoactive site of the ROP5 pseudokinase domain mediates its effect on Toxoplasma virulence. Journal of Biological Chemistry. 2011 Aug 19;286(33):29366-75.

38. Reese ML, Shah N, Boothroyd JC. The Toxoplasma pseudokinase ROP5 is an allosteric inhibitor of the immunity-related GTPases. Journal of Biological Chemistry. 2014 Oct $3 ; 289(40): 27849-58$.

39. Sanchez CP, Stein WD, Lanzer M. Dissecting the components of quinine accumulation in Plasmodium falciparum. Molecular microbiology. 2008 Mar;67(5):1081-93.

40. Secrieru A, Costa IC, O'Neill PM, Cristiano ML. Antimalarial Agents as Therapeutic Tools Against Toxoplasmosis-A Short Bridge between Two Distant Illnesses. Molecules. 2020 Jan;25(7):1574.

41. Shammaa AM, Powell TG, Benmerzouga I. Adverse outcomes associated with the treatment of Toxoplasma infections. Scientific reports. 2021 Jan 13;11(1):1-8.

42. Shiojiri D, Kinai E, Teruya K, Kikuchi Y, Oka S. Combination of clindamycin and azithromycin as alternative treatment for Toxoplasma gondii encephalitis. Emerging infectious diseases. 2019 Apr;25(4):841. 
43. Sommerville C, Richardson JM, Williams RA, Mottram JC, Roberts CW, Alexander J, Henriquez FL. Biochemical and immunological characterization of Toxoplasma gondii macrophage migration inhibitory factor. Journal of Biological Chemistry. 2013 May 3;288(18):12733-41.

44. Syn G, Anderson D, Blackwell JM, Jamieson SE. Toxoplasma gondii infection is associated with mitochondrial dysfunction in-vitro. Frontiers in cellular and infection microbiology. 2017 Dec 12;7:512.

45. Willmott CJ, Critchlow SE, Eperon IC, Maxwell A. The complex of DNA gyrase and quinolone drugs with DNA forms a barrier to transcription by RNA polymerase. Journal of molecular biology. 1994 Sep 29;242(4):351-63.

\section{CONFLICT OF INTEREST}

The authors declare that the research was conducted in the absence of any commercial or financial relationships that could be construed as a potential conflict of interest. 


\section{Figure 1}

DHQ(red) bonded to TgDNA gyrase in the binding pocket

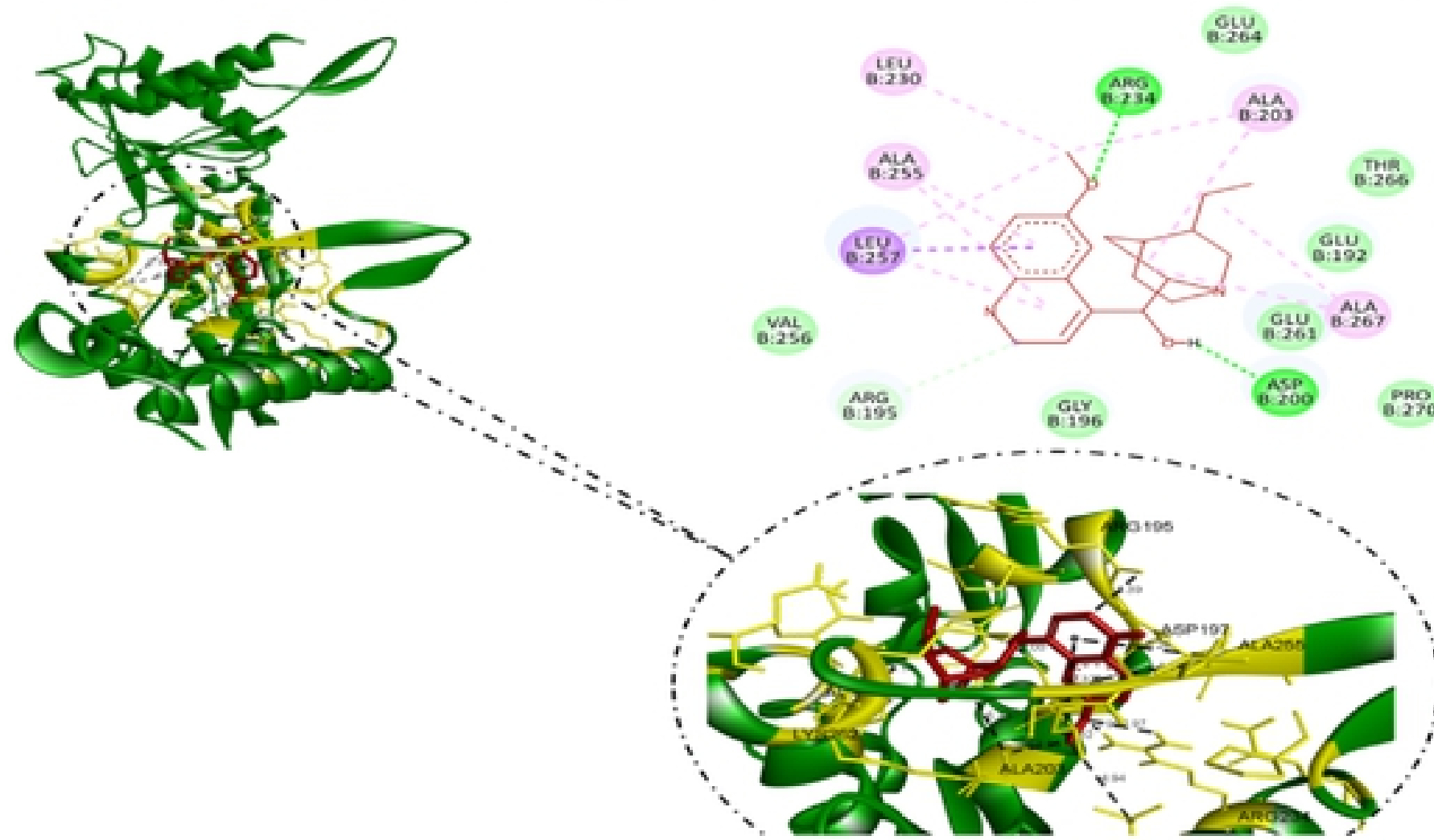

Interactions

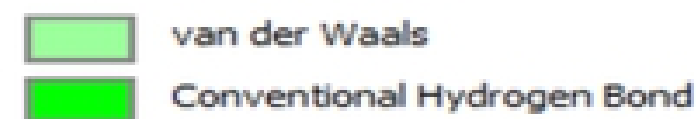

Conventional Hydrogen Bond

Carbon Hydrogen Bond

Pi-Sigma

Alkyl

Pi-Alkyl

As shown in Figure 1, DHQ forms a strong hydrogen bond with ARG234 producing a bond distance of $1.90 \AA$, for which DHQ acts as the hydrogen acceptor and ARG234 the donor. The ALA255 of Tg DNA gyrase binds to DHQ via hydrophobic interactions, forming a pi-alkyl bond with DHQ with a bond distance of $4.69 \AA$ in the binding pocket. Forming hydrogen bond at $2.97 \AA$ with DHQ, TgDNA gyrase via ARG234 acts as hydrogen donor for which DHQ is the acceptor. DHQ is able to form a stable hydrogen bond with TgDNA gyrase via the gyrase's ASP200 amino acid residue which acts as a hydrogen acceptor. A bond distance of $1.99 \AA$ is established between them. ARG195 interacts with DHQ via hydrogen bond also, for which ARG195 is the hydrogen acceptor. LEU257 of TgDNA gyrase establishes pi-sigma interaction with DHQ to establish a hydrophobic bond of distance $3.38 \AA$. Other hydrophobic bonds formed by TgDNA gyrase with DHQ established in the binding pocket includes amino acids ALA203 (bond distance, $3.55 \AA$ ), ALA255 (bond distance, $5.26 \AA$ ), LEU257 (bond distance, $5.32 \AA$ ), ALA267 (bond distance, $4.72 \AA$ ), LEU230 (bond distance, $4.94 \AA$ ), LEU257 (bond distance, $4.62 \AA$ ) and ALA255 (bond distance, $4.69 \AA$ ). 
Figure 2 DNA gyrase $\mathbf{A}$ of Mycobacterium tuberculosis
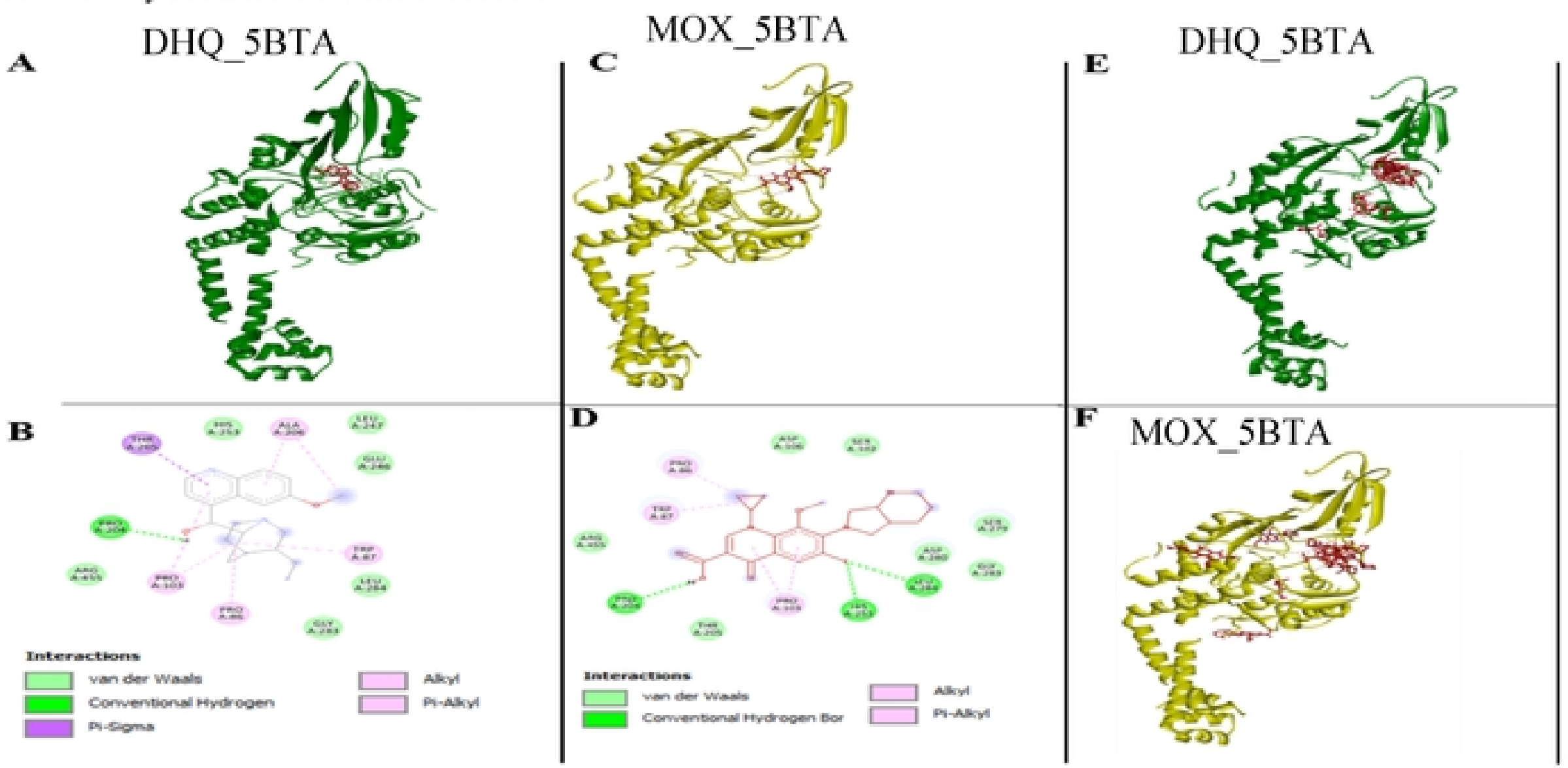

Figure 2A, 2B, 2E shows DHQ interaction with gyrase A (5BTA) (a topoisomerase II complex of Mycobacterium tuberculosis H37Rv). As shown in Figure 2A, the 3D rendition of DHQ in red color interacting with gyrase $\mathrm{A}$ in green color. Figure $2 \mathrm{D}$ is a $2 \mathrm{D}$ depiction of DHQ interacting with gyrase at the binding pocket indicates that proline at amino acid position 204 interacts directly with DHQ via hydrogen bonding, whereas PRO86 and PRO103 shows alkyl interaction with the compound. TRP87, and ALA206 also shows alkyl and pi-alkyl interaction with DHQ. A single pi-sigma bond is formed between THR205 and DHQ.

Figure 2C depicts Moxifloxacin (red) bound to gyrase A (yellow), whereas Figure 2D shows a 2D rendition of moxifloxacin (red) interacting with the binding pocket atoms of gyrase A via 3 hydrogen bonds and four alkyl and pi-alkyl bonds.

Figures $2 \mathrm{E}$ and $2 \mathrm{~F}$ also shows all the 9 different conformational poses of the DHQ (in red) bonding to gyrase A molecule in green and the 9 different poses of moxifloxacin (red) in the binding pockets of gyrase (yellow) respectively. 


\section{Figure 3}

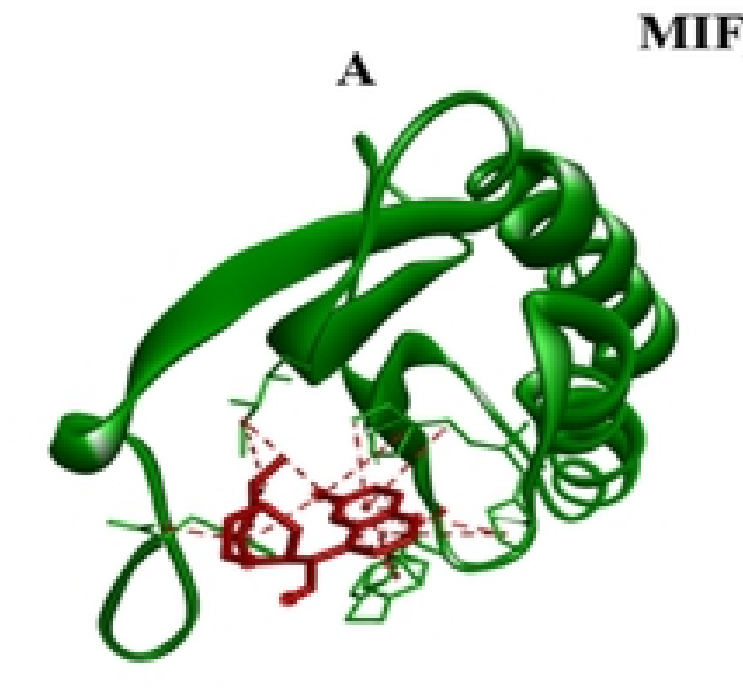

MIF_DHQ

B

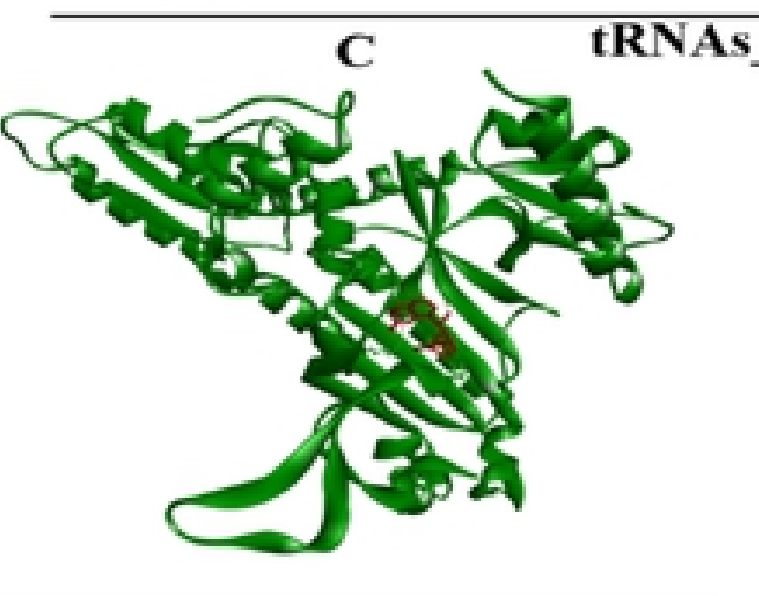

DHQ D
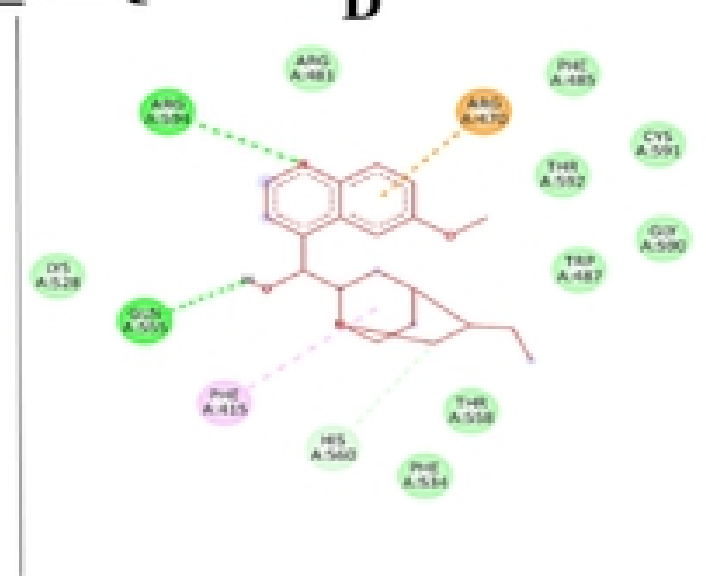

Interactions

$\square$ van der Waals

Conventional Hydrogen Bond

Carbon Hydrogen Bond

Pi-Cation

Pi-Alkyl

Figure 3 shows DHQ binds stably to T. gondii MIF. As shown in Figure 3A, DHQ is shown bound to two loops of MIF molecule. The 2D rendering (Figure 3B) shows that the most stable conformation was achieved by the binding of DHQ to the PHE114, PRO1, ILE65, LYS33, TYR37, and PRO34 via hydrogen bonding, alkyl, pi-alkyl, pi-cation, pi-pi stacked, and alkyl interactions respectively.

Figure 3C is the 3D depiction of the DHQ interacting with tRNA synthetase of $T$. gondii. Whereas Figure 3D, a $2 \mathrm{D}$ rendition shows that DHQ binds to tRNA synthetase of $T$. gondii via two hydrogen bonds at amino acids ARG594, and GLN555. There exists also a van der Waals force between the compound and the amino acid HIS560, a pi-alkyl interaction at amino acid PHE415 and pi-cation interaction with ARG470. 
Figure 4

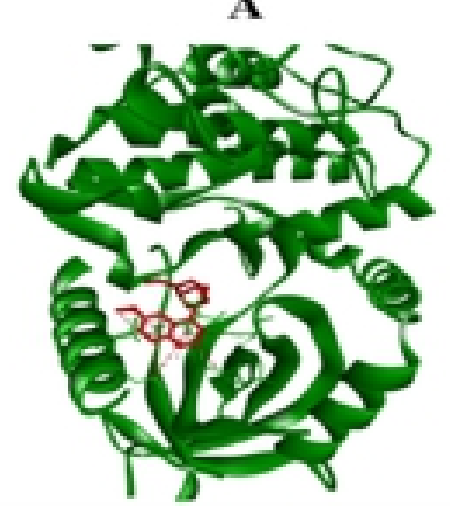

ROP5B_DHQ

B

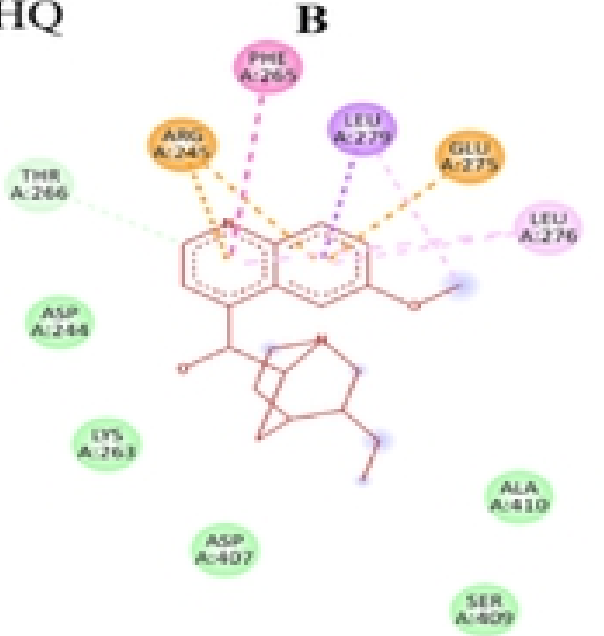

Interactions

$\square$ van der Waals

$\square$ Carben Hydrogen Bond

P-Cason

A.Arion

A.sioms

Aknt

A.Akyl
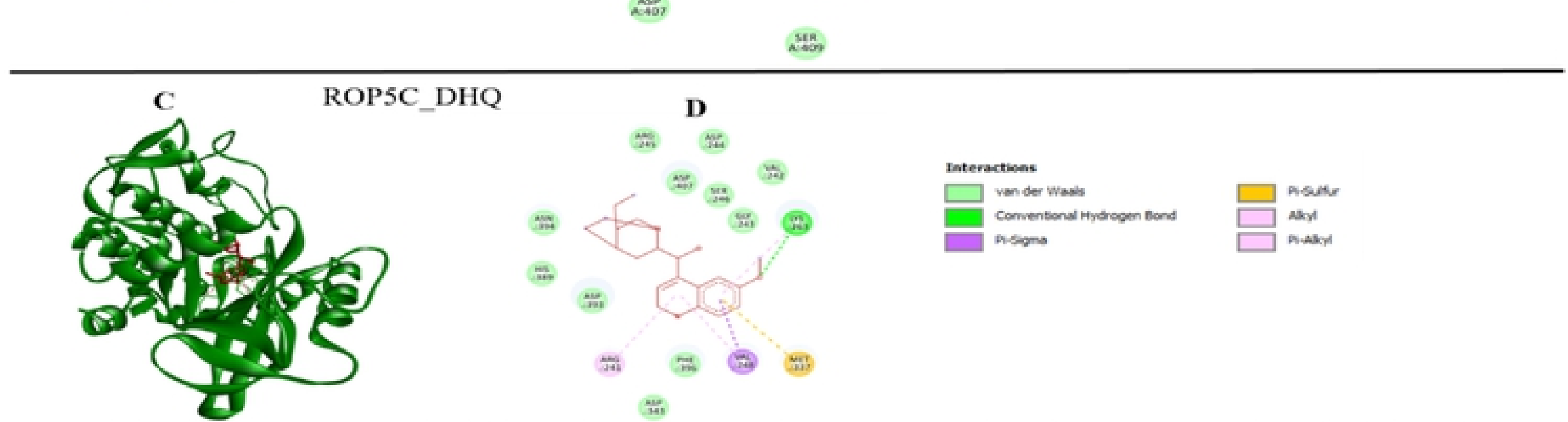


\section{Figure 5}

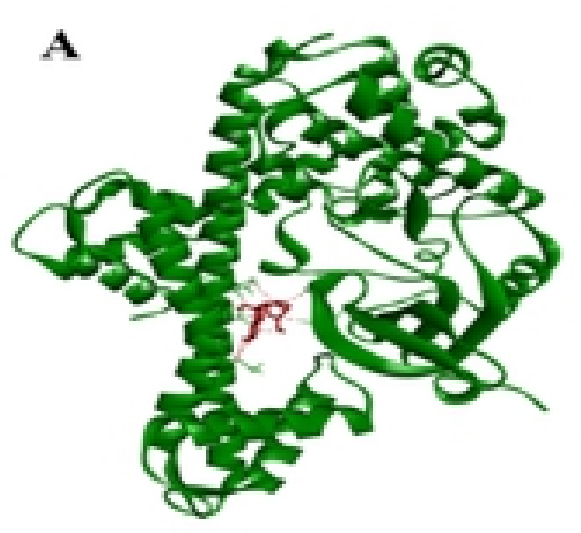

\section{TgCDPK1_DHQ}

| B

B 1250

(4⿻)丨

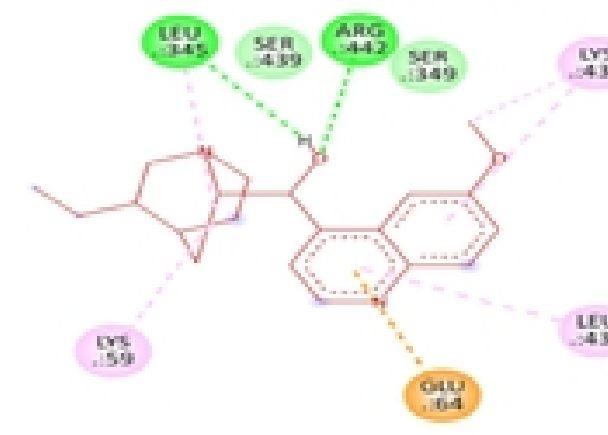

Interactions

van der Waals

Conventional Hydrogen Bond

Pi-Arion

Alkyl

Pi-AlkyI
C

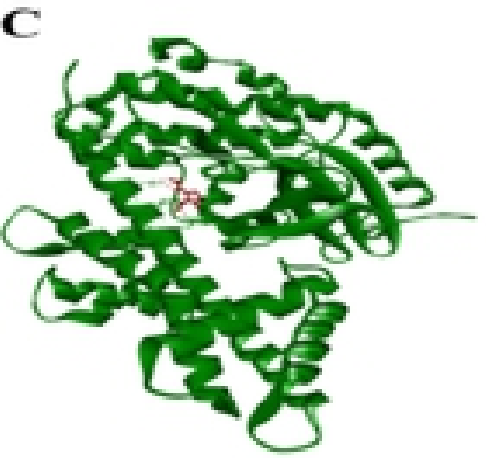

TgCDPK1_3-carboxamide

\section{D}

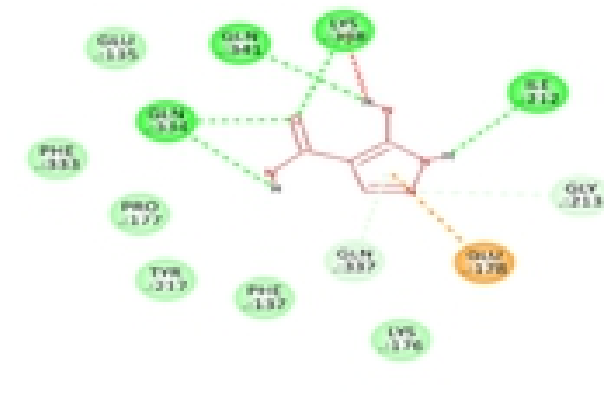

Interactions

$\square$ van der Wavk

Conventional Hydrogen Band

Carbon Hydropen Bond

Untavorable Donor-Donor A.Amion

A-Donor Hydrogen Bond

Figure $\mathbf{5 A}$ shows the $3 \mathrm{D}$ rendition of DHQ-TgCDPK1 interaction. Figure 5B shows a 2D diagrammatic illustration of DHQ interacting with TgCDPK1 via two hydrogen bonds emanating from LEU345 and ARG439, two alkyl bonds formed between Lys59, LYS435 and LEU438 and finally a pi-anion interaction between GLU64 and DHQ.

In Figure 5C, 3-Amino-1H-pyrazole-4-carboxamide is depicted interacting with TgCDPK1, and Figure 5D shows the 2D rendition of the interaction which emanates from the binding of the compound to the active site of TgCDPK1 at the following residues; ILE212, LYS338, GLN334, GLN341, and GLU178 which all demonstrated conventional hydrogen bonding with the compound except GLU178 which established a pi-anion bond with 3-Amino-1H-pyrazole-4-carboxamide. LYS338 however shows a strong unfavorable donor-donor interaction with the hydrogen moiety of 3-Amino-1H-pyrazole-4-carboxamide. This interaction therefore caused a considerable loss of affinity of 3-Amino-1H-pyrazole-4-carboxamide to TgCDPK1 compared to DHQ. 


\section{Figure 6}

A DHQ(red) bonded to SOD3 residues in the binding pocket

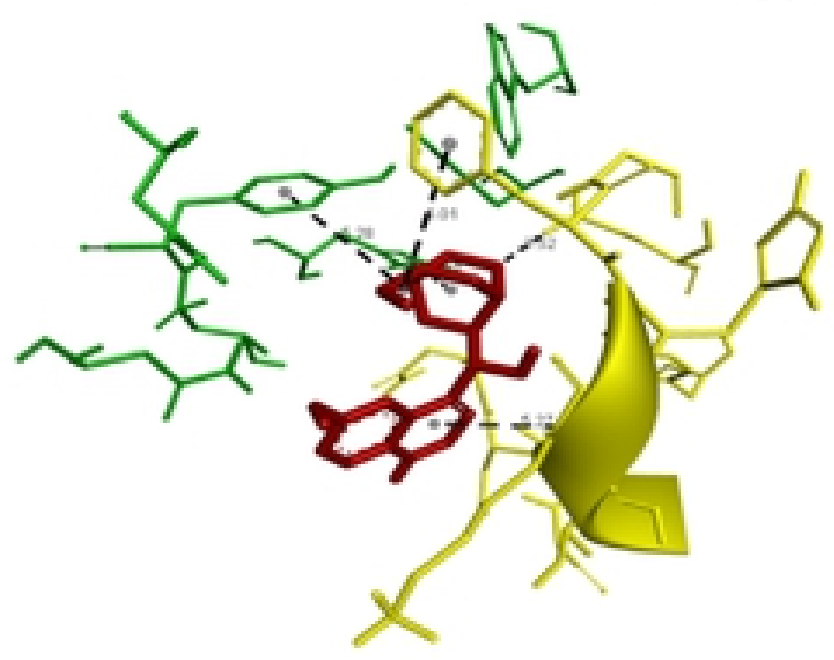

C DHQ(red) bonded to SOD2 residues in the binding pocket

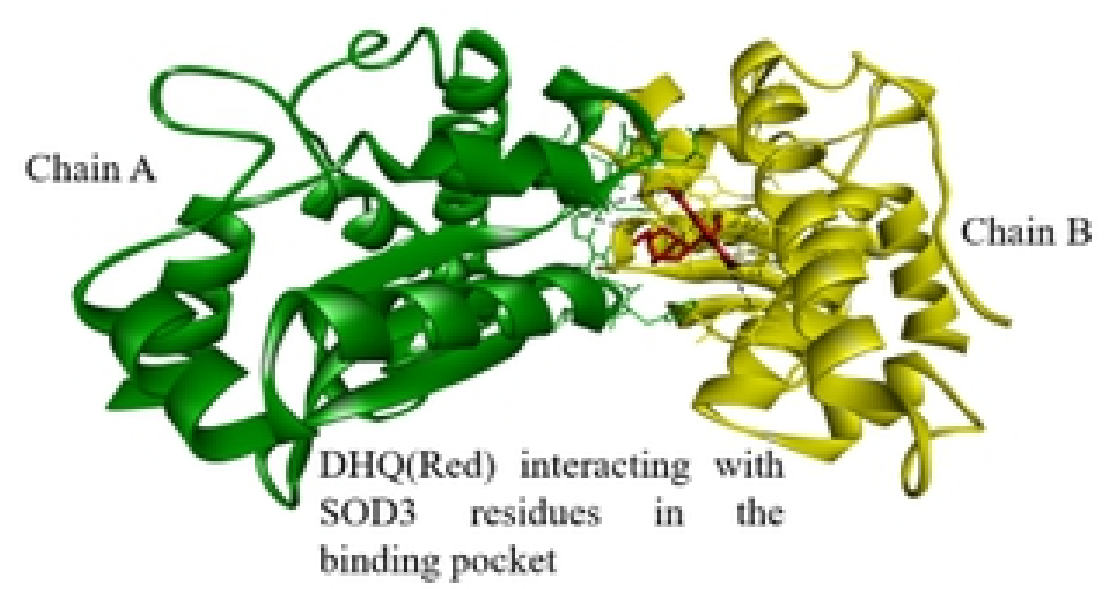

B 2D display of DHQ SOD3 interaction

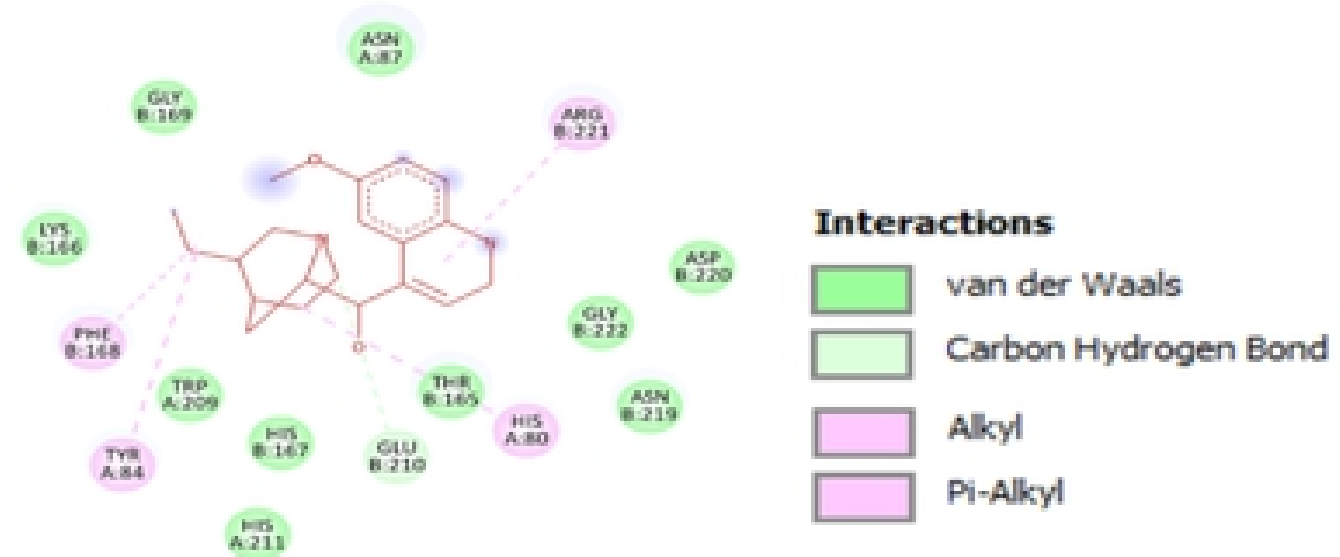

D 2D display of DHQ_SOD2 interaction $\cos _{2 \rightarrow 3}$

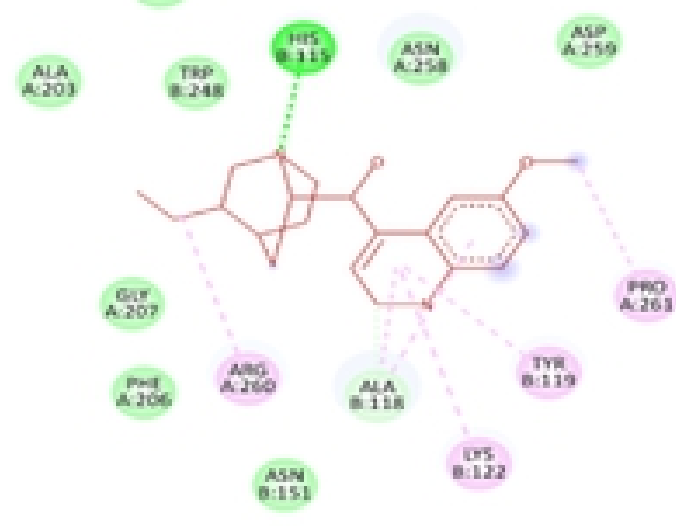

\section{Interactions}

Conventional Hydrogen Bond Carbon Hydrogen Bond

Alkyl

Pi-Alkyl 
In Figure 6A and 6B depicts DHQ (red) bound to SOD3 molecule in the binding pocket. As shown in the Figure 6B, DHQ establishes stable hydrogen bond with GLU210. It also interact with SOD3 via alkyl and pialkyl hydrophobic interactions through amino acids HIS80, TYR84, PHE168, and ARG221. Other weaker van der Waals interactions established between SOD3 and DHQ were recorded on amino acids LYS166, GLY169, ASN87, THR165, HIS167, TRP 209, HIS211, ASN 219, ASP220 and GLY222. Together these interactions enhanced the stability of the binding of DHQ to SOD3 molecule.

The Figure 6C and 6D depicts the interaction of DHQ (red) with SOD2 molecule. As shown in the Figure 6C, DHQ formed a stable hydrogen bond with ALA118 and HIS115. It also interact with SOD2 via alkyl and pi-alkyl hydrophobic interactions through amino acids LYS122, TYR119, ARG260, and PRO261. The weak van der Waals interactions established between SOD2 and DHQ were recorded on amino acids ASN151, ALA203, PHE206, GLY207, TRP248, GLU249, ASN258, and ASP259. Accumulatively these interactions boosted the stability of the binding of DHQ to SOD2 molecule. 
Figure 7

A DHQ (yellow) bound to Chain $\mathrm{A}$ of $\mathrm{Tg}$ catalase

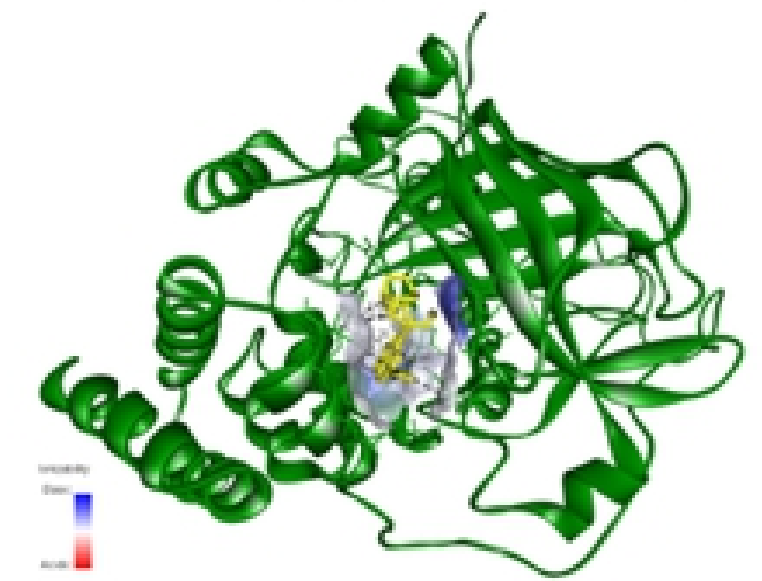

C DHQ (red) bound to Chain A of Tg peroxidoxin

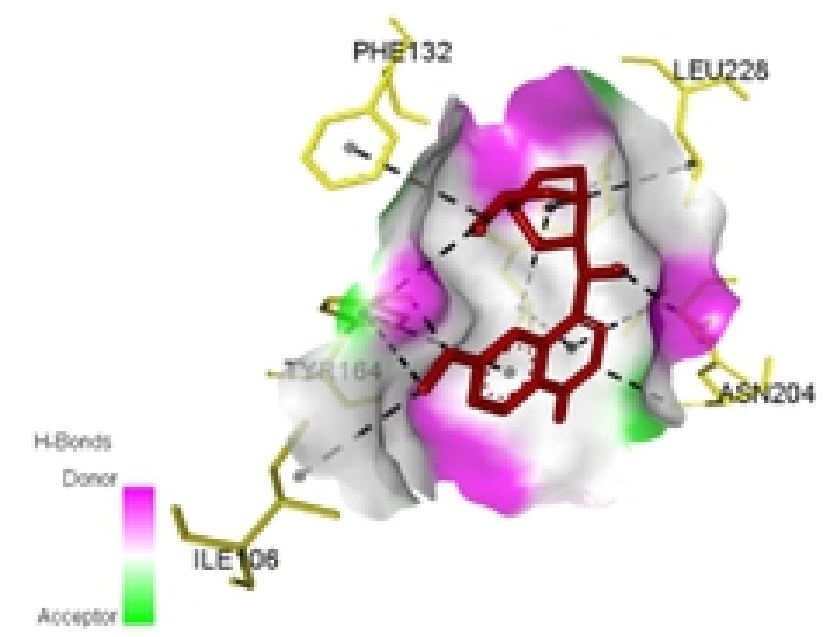

B 2D display of DHQ interaction with $\mathrm{Tg}$ catalase in the binding pocket

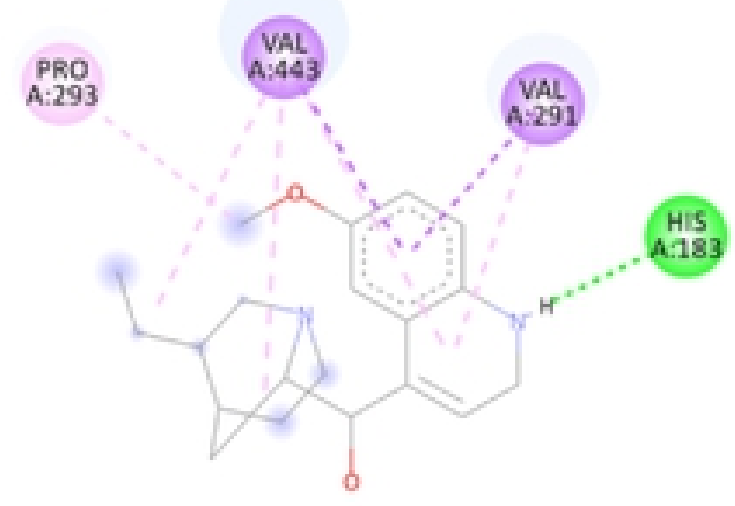

Interactions

$\square$ van der Waals

Conventional Hydrogen Bond

Pi-Sigma

Alkyl

D 2D display of DHQ interaction with $\mathrm{Tg}$ peroxidoxin in the binding pocket

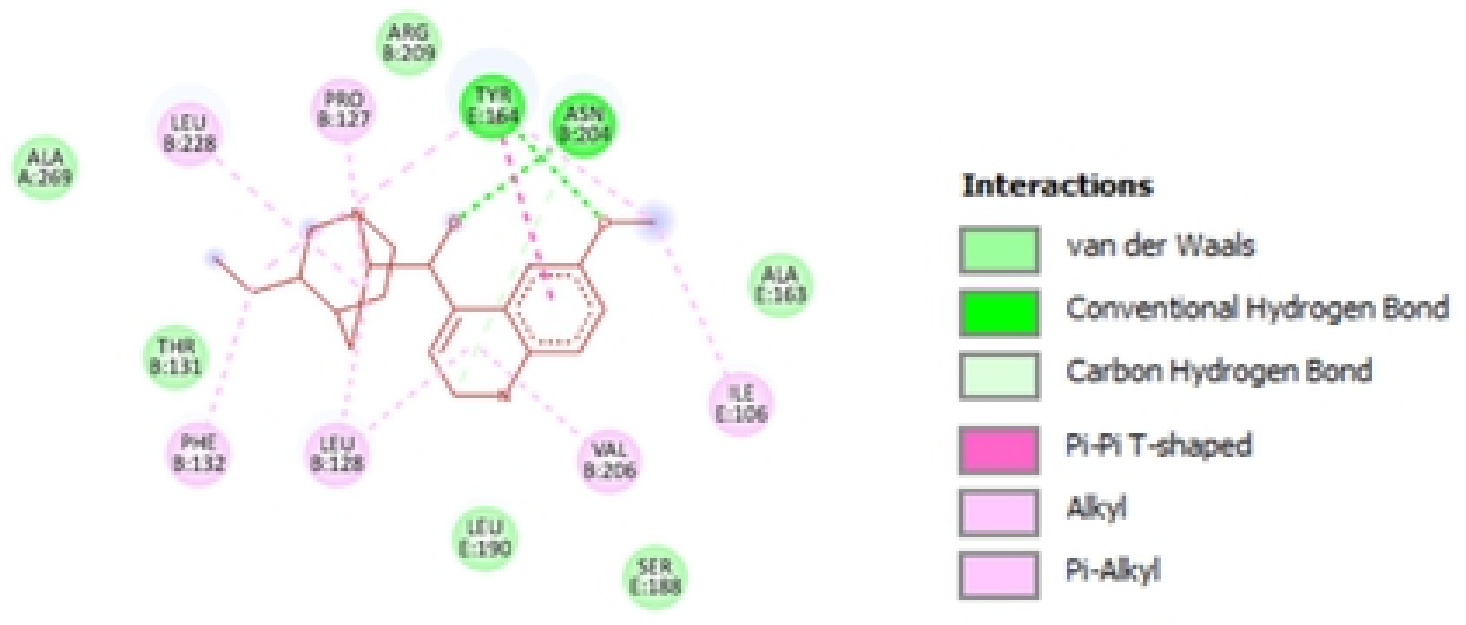


In Figure 7A and 7B depicts DHQ (red) bound to Tg Catalase chain A. As depicted in the Figure 7B, DHQ forms stable hydrogen bond with HIS183, and shares a pi-sigma bond with VAL291 and VAL443 respectively. VAL291 and VAL443 are able to also interact with DHQ via alkyl hydrophobic interactions. Tg Catalase also is able to bind to DHQ via its PRO293 by another hydrophobic alkyl interaction with the hydroxyl group of the DHQ.

The Figure 7C and 7D depicts the interaction of DHQ (red) with Tg peroxidoxin molecule. As shown in the Figure 7D, Tg peroxidoxin binds tightly to DHQ via two hydrogen bonds established between DHQ at amino acids AS204 and TYR164. It is also able to stabilize DHQ in the binding pocket via hydrophobic interactions such as pi-pi-T shaped interactions, alkyl and pi-alkyl interactions through the amino acids TYR164, IL3106, VAL206, LEU128, PHE132, LEU228 and PRO 127. Also, a weaker van der Waal force exist between ALA163, SER188, LEU190, THR131 and ALA269 with DHQ. These altogether stabilized DHQ in Tg peroxidoxin binding pocket. 